\title{
The Effect of Filler Orientation and Distribution on the Electrical and Mechanical Properties of Graphene Based Polymer Nanocomposites
}

\author{
P. K. Rajamani ${ }^{1}$, R. Boros ${ }^{1}$ \\ ${ }^{1}$ Budapest University of Technology and Economics, Department of Polymer \\ Engineering, Müegyetem rkp. 3., 1111 Budapest, Hungary \\ e-mail: rajamani@pt.bme.hu
}

Abstract: The aim of my proposed study is to develop an alternative production technique of graphene via in-situ exfoliation of graphite in the hosting polymer matrix. The production process is carried out by an alternative top-down production technique for graphene-based polymer nanocomposites called 'pressing and folding' (P\&F), via in situ exfoliation of expanded graphite (EG) inside the hosting linear lowdensity polyethylene (LLDPE) matrix. In this way, the properties of the samples containing different wt \% of EG is studied as a function of $\mathrm{P} \& \mathrm{~F}$ cycles, corresponding to EG exfoliation and distribution throughout the matrix volume. The results confirm that the EG particles were exfoliated completely and increasingly distributed in LLDPE with the number of cycles, and mainly oriented on the plane of the samples. This find was confirmed by a low in-plane resistivity was found for samples prepared between 50 and 150 cycles.

Keywords: In-situ exfoliation, Graphene, Polyethylene, Polymer Nanocomposites, Mechanical Properties

\section{Introduction}

Graphene is a single atom thick sheet of $\mathrm{sp} 2$-hybridized carbon atoms [1]. There has been a rapid rise of interest in the study of the structure and properties of graphene following the first report in 2004 of the preparation and isolation of single graphene layers in Manchester [2]. A single-layer graphene had previously considered as an academic material which would not be possible to remove from the graphite since such 2D crystals would be unstable thermodynamically and/or might roll up into scrolls if prepared as single atomic layers [3]. Research upon the material has now broadened considerably as it was soon realized that graphene might have 
other interesting and exciting physical properties such as high levels of stiffness and strength, and thermal conductivity, combined with an impermeability to gases. One obvious application of graphene is in the field of nanocomposites [4] [5] [6] and it has been noticed that the modification of graphene with polymers and the fabrication of graphene-based nanocomposites attracted more and more interests recently.

Polymer based graphene nanocomposites show superior mechanical, thermal, electrical and gas barrier properties when compared to the neat polymer [7] [8] [9] [10]. The most important aspect of these nanocomposites is that all these improvements are obtained at very low filler loadings in the polymer matrix [11] [12]. However, the improvement of these properties depends on the distribution and dispersion of graphene layers in the polymer matrix and as well as interfacial bonding between them. For the efficient utilization of graphite as filler in a polymer composite, its layers must be separated and dispersed throughout the polymeric matrix. Graphite nanoplatelet (GNP), the basic unit obtained by exfoliation of the natural graphite and having a platelet thickness varying from less than 0.34 to 100 $\mathrm{nm}$, is a promising low cost and lightweight alternative to other carbon-based electrically conductive reinforcements for polymer composites [13]. Mainly, two different types of GNPs, such as expanded graphite (EG) and graphite oxide (GO) have been widely used to produce conducting nanocomposites along with improved mechanical properties [14]. There are many studies on these GNP composites based on a range of polymers, including epoxy [15], PMMA [16], polypropylene [17], polyethylene [18] [19], polystyrene [20], nylon [21] and silicone rubber [22].

However, several studies indicated that the dispersion of graphene particles in polymer matrices become challenging due to the interfacial incompatibility [23] of the constituents and for this reason, selection of an appropriate manufacturing technique is essential. There have been various studies discussing the methods of fabricating graphene-based polymer nanocomposites and their corresponding influence on materials properties. For example, Istrate et al [24] prepared a polymer nanocomposite with polyethylene terephthalate (PET) as a matrix and it was reinforced with EG layers solely via melt blending. They have found that with a very little nanofiller loading of $0.07 \mathrm{wt} \%$, the material showed an increase in the elastic modulus higher than $10 \%$ and an enhancement in the tensile strength of more than $40 \%$ when compared to pure PET.

Another important fabrication method was investigated by Tang et al [25], where they fabricated GO/epoxy composites via solution blending. They prepared different dispersions of GO sheets with and without ball mill mixing and proved that a better GO dispersion in epoxy matrix was obtained after using the ball mill mixing process. Other commonly used fabrication technique includes in-situ polymerization [26] [27], Latex mixing [28] and electropolymerization [29]. Among 
the discussed fabrication methods, the solution blending is much better for producing quite homogeneous graphene-based polymer composites because the polymer and the graphene are dissolved in the same or miscible solvent. Also, in-situ polymerization and latex mixing can be a good alternative method for generating homogeneous composites but agglomeration of graphene layers inside the polymer matrix can be a common problem. So, to understand and obtain better graphene dispersion and less agglomeration, there is a need to develop an alternative and simple fabrication technique.

In this present work, a significant effort has been directed to the alternative fabrication of LLDPE/EG nanocomposites and investigate their mechanical and electrical properties. For that purpose, LLDPE are mixed with expanded graphite in a hot press and subjected to 'pressing and folding' ( $\mathrm{P} \& \mathrm{~F})$. This work had started with the testing of 10, 20, 50, 100, 150, 200 and 500 cycles of $0.5 \mathrm{wt} \%$ of EG content. Later, the experiments are conducted with much higher EG content of 5, 10, 16 and $56 \mathrm{wt} \%$. The main goal is to assess the influence of number of cycles and the filler content on the tensile properties of the prepared polymer nanocomposites. The second part of the manuscript involves in finding the percolation threshold, where the polymer nanocomposites starts to conduct electricity. For this study, four samples with each cycle are made from hot press are tested with the help of twopoint probe method: two for in-plane conductivity and two for out-of-plane conductivity. The third part was to examine the micro-structures of the prepared samples. The samples are analysed in SEM separately with two different broken samples: in tensile machine and cryogenically broken. The final section offers future prospects and conclusions.

\section{Materials and Methods}

\subsection{Materials Used}

The polymer matrix used in this process is linear low-density polyethylene called Flexirene ${ }^{\circledR}$ MS20A (LLDPE). LLDPE is a commodity polymer, cheap, versatile, with widely uses in a variety of forms. The Graphene Intercalation compound (GICs), commonly called Expanded Graphite is used as a reinforcement of the LLDPE. The Timerex ${ }^{\circledR}$ C-THERM 002 is the particular specification of EG which is being used. EG will improve the properties of the polymer matrix, which is directly related to the degree of dispersion of the nanofillers in the polymer matrix. 


\subsection{Fabrication of polymer nanocomposites}

This work takes advantage of an alternative top-down production technique for graphene-based polymer nanocomposites, called "pressing and folding" (P\&F), via in situ exfoliation of graphite inside the hosting polymer matrix. In particular, linear low-density polyethylene Flexirene ${ }^{\circledR}$ MS20A (LLDPE) and expanded graphite Timerex ${ }^{\circledR}$ C-THERM ${ }^{\mathrm{TM}} 002$ (EG) were joined at first, and then gradually mixed by pressing \& folding the samples inside a hot-press many times as shown in Fig. 1.

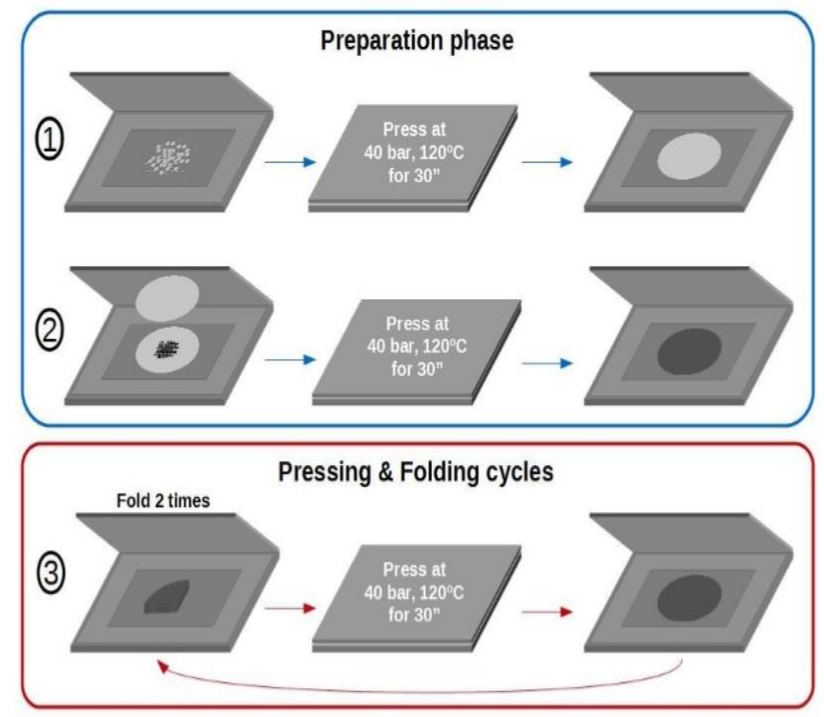

Figure 1. Preparation of samples by pressing and folding

Initially, $1.4 \mathrm{~g}$ (0.72 $\mathrm{g}$ for each discs) of LLDPE discs are prepared using the hot press with the elevated temperature of $120^{\circ} \mathrm{C}$ with the pressure of $40 \mathrm{bar}$ and for the duration of 30 seconds. Before incorporating EG into the LLDPE, the pristine LLDPE sample is saved for the benchmark. At first, the minimum filler content of $0.5 \mathrm{wt} \%$ to be added between the two PE discs and hot pressed again. After the first cycle the PE plated are folded two times as shown in Fig. 1 and again the sample is hot pressed. Throughout the process, the temperature, Pressure and the press time duration should be kept constant for uniform dispersion of EG. This pressing and folding cycle is continued for different desired cycles. This work had started with the testing of $10,20,50,100,150,200,500$ cycles of $0.5 \mathrm{wt} \%$ of EG content. Later, the experiments are conducted with higher EG content of $5 \mathrm{wt} \%, 10 \mathrm{wt} \%, 16 \mathrm{wt} \%$ 
and $56 \mathrm{wt} \%$ (Fig. 2). The hot press configuration pressure of 40bar is applied at a same value for every cycle so that the final sample will have an equal thickness. After the pressing and folding cycles are finished for all the filler content, five dumbbell specimens are made corresponding to ASTM D 638-02a.
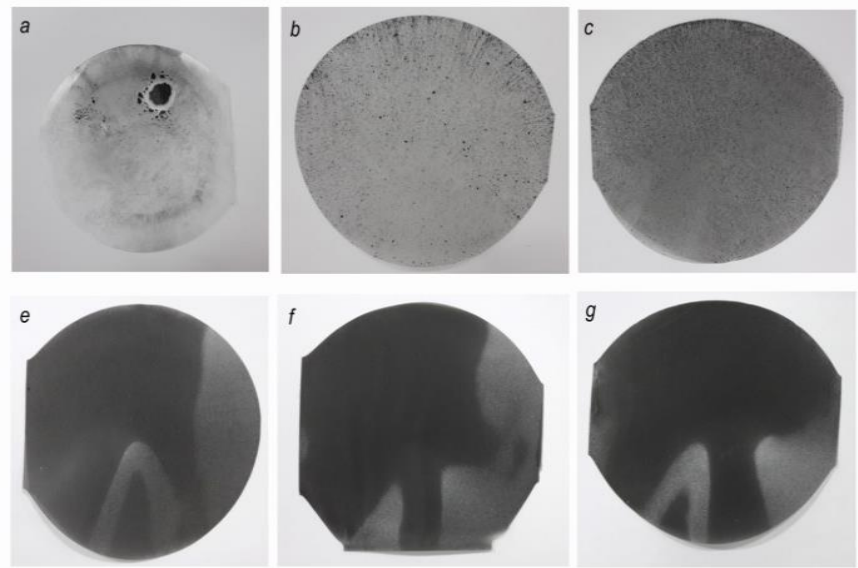
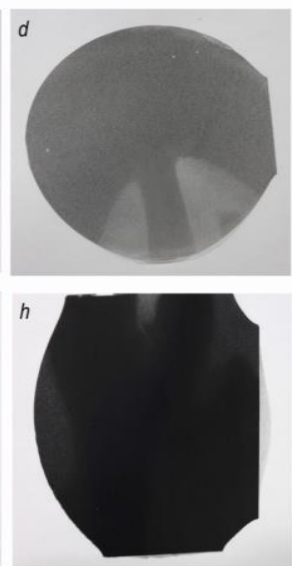

Figure 2. Prepared LLDPE / 0.5 wt\% EG samples after a) 1 cycle b) 10 cycles c) 20 cycles d) 50 cycles e) 100 cycles f) 150 cycles g) 200 cycles h) 500 cycles

\subsection{Characterization Techniques}

The 'dumbbell' shaped specimen is loaded in the $1 \mathrm{kN}$ load cell of the Instron. As the specimen has a very low thickness $(\sim 0.230 \mathrm{~mm})$, the tensile grips should be used to ensure that the specimen is tightly held to the load cell. The rate of speed for the specimen is maintained always at $1 \mathrm{~mm} / \mathrm{min}$. With this test, the following parameters can be obtained, such as: tensile strength, elongation at yield, elongation at break, Modulus of elasticity, nominal strain.

The electrical conductivity of the specimen can be obtained with the help of the two-point probe method. From the sample made from the hot press, a strip of approximately $12 \times 8 \mathrm{~mm}$ of the specimen is trimmed to carry out the electrical testing. For each cycle, four strips are trimmed: two for the in - plane conductivity and two for the out-of-plane conductivity. The strips are individually placed between an aluminium coil prepared, which acts as the extremity. The silver paste can be used between the specimen and the aluminium coil, to keep the aluminium in place and to have an unaffected electric flow between the extremities. 
The micro-structures of the prepared nanocomposites were studied analysing the cross-sections of cryogenically broken samples by SEM. The samples were analysed separately with two different broken samples: tensile and cryogenically broken. First, the samples broken using the Instron are analysed through the SEM. The other type of sample can be broken cryogenically with the help of liquid nitrogen. The samples should be sputter coated with conducting material like gold or palladium alloy before introducing in the SEM. This coating is needed to prevent charging of a specimen with an electron beam introduction in conventional SEM.

\section{Results and Discussion}

This section analyses the effect of EG filler content on the LLDPE samples and number of pressing and folding cycles. The section was divided into three parts. The first part and second part are devoted to the mechanical and electrical characterization of the prepared samples, respectively. The last part investigates the dispersion and distribution of EG and exfoliation of graphene in polymer matrix with the help of SEM.

\subsection{Mechanical Characterization}

For the minimum filler content of $0.5 \mathrm{wt} \%$, there are no significant changes were noted to the elastic modulus of pure LLDPE up to 200 cycles, thus the value at 1 cycle $(135 \pm 5 \mathrm{MPa})$ was taken as reference. The reinforcement due to the EG as a function of cycles can be evaluated by dividing the elastic modulus of each composite by the reference modulus of LLDPE as shown in Fig.3. It was found that the reinforcement $(\mathrm{Ec} / \mathrm{Em})$ does not show any proper pattern with the number of increasing cycles.

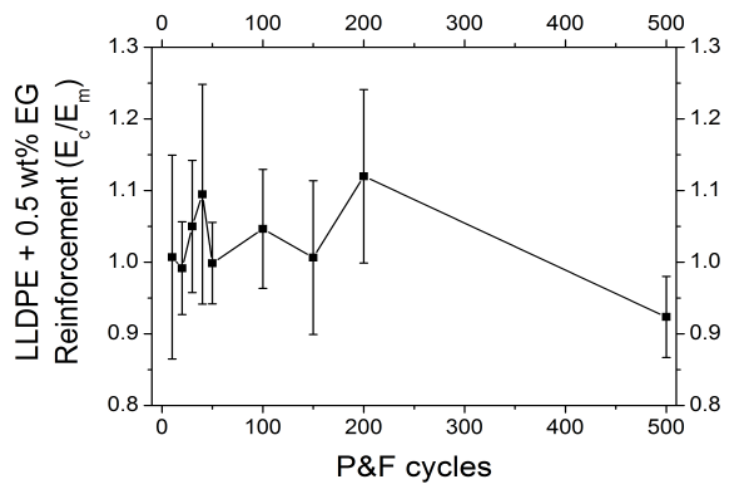

Figure 3. Reinforcement found for sample of LLDPE/ $0.5 w t \%$ EG as a function of $P \& F$ cycle 
This non-uniformity in the reinforcement is due to the very small amount of filler that has been added to the LLDPE polymer. So that maintaining an exact 0.5 wt $\%$ is a big challenge. The sudden drop in the reinforcement for the 500 cycle is the actual filler content found to be only $0.3 \mathrm{wt} \%$ rather than $0.5 \mathrm{wt} \%$. It was expected that the 500-cycle composite will have the highest reinforcement but because of the long process of 500 cycles in the hot press and also because of the small EG content filled in the LLDPE, it does not show a proper pattern (Fig 4). But it's not the case for the LLDPE/ $10.7 \mathrm{wt} \% \mathrm{EG}$ and LLDPE/16 wt\% EG.

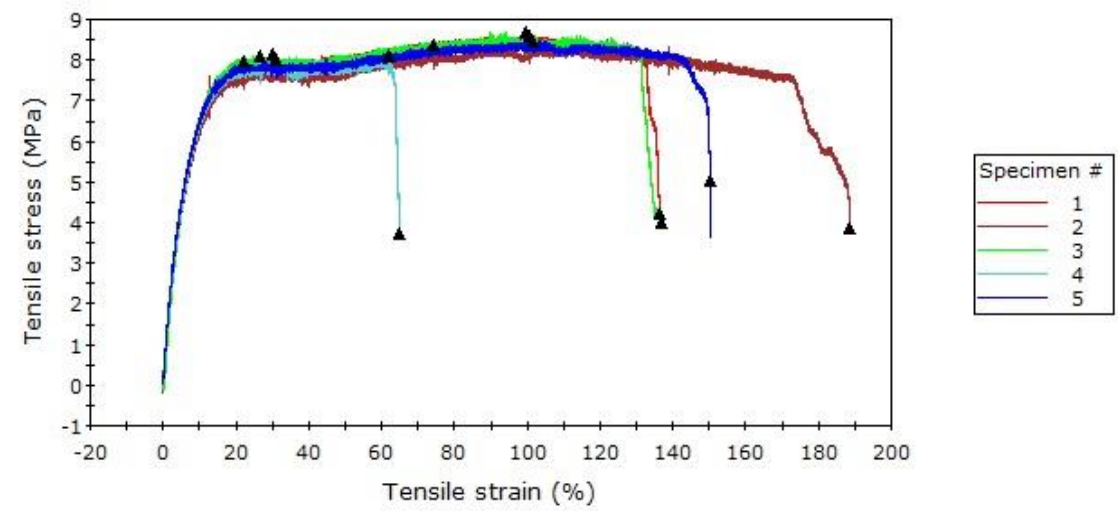

Figure 4. Tensile stress Vs strain for the LLDPE / 0.5 wt \% EG at 500 P\&F cycles.

Although all nanocomposites exhibit higher modulus than pure LLDPE, they show final failure at much lower stress and strain than the pure LLDPE. In general, the improvement of elastic modulus is attributed to the good dispersion of nanosized particles and good interfacial adhesion between the EG filler and the LLDPE matrix. So that the mobility of polymer chains is restricted under loading. The lower tensile strength of nanocomposites could be due to number of reasons such as weak interfacial bonding between the EG and matrix interfaces, aggregates of graphite nanosheets and nano to micro size process related defects. Fig. 5 shows the stress at break and strain at break as a function of $P \& F$ cycles and also the yield stress and strain as a function of $P \& F$ cycles. Therefore, the mechanical property of LLDPE / $0.5 \mathrm{wt} \%$ EG does not show a good improvement in the P \& F technique. It also showed that solution intercalation method shows better results than the melt mixing. As the matrix used here is a thermoplastic polymer (LLDPE), therefore melt mixing along with the new technique called $P \& F$ technique was carried out. 

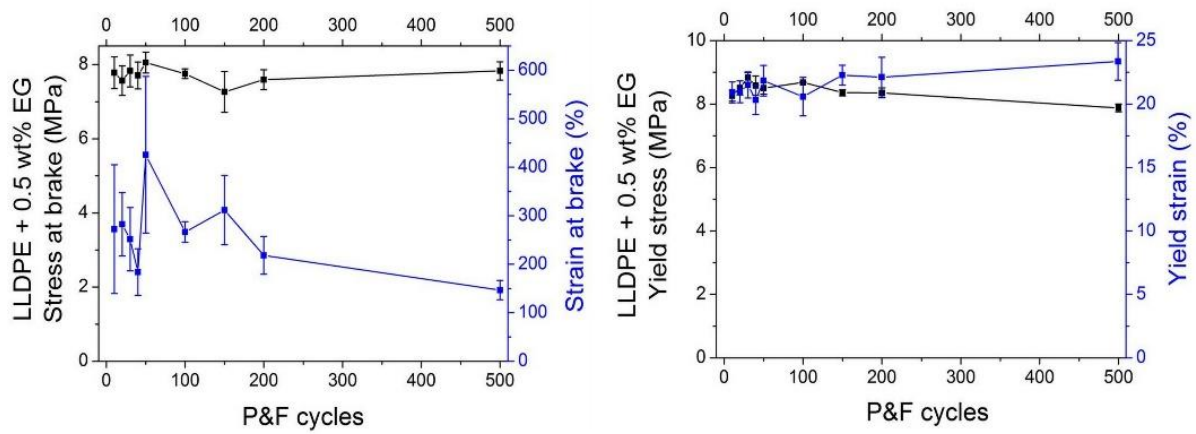

Figure 5. As a function of $P \& F$ cycles for the LLDPE / 0.5 wt\% EG Nanocomposites a) Stress and strain at break b) Yield stress and Yield strain

The young's modulus is improved than the $0.5 \mathrm{wt} \%$ for the 200 cycles. As there were no change in the lower P \& F cycles $(10,20,100,150)$, only 200 cycles technique is performed for the $5 \mathrm{wt} \%$. The tensile stress vs the tensile strain is shown in the Fig. 6.

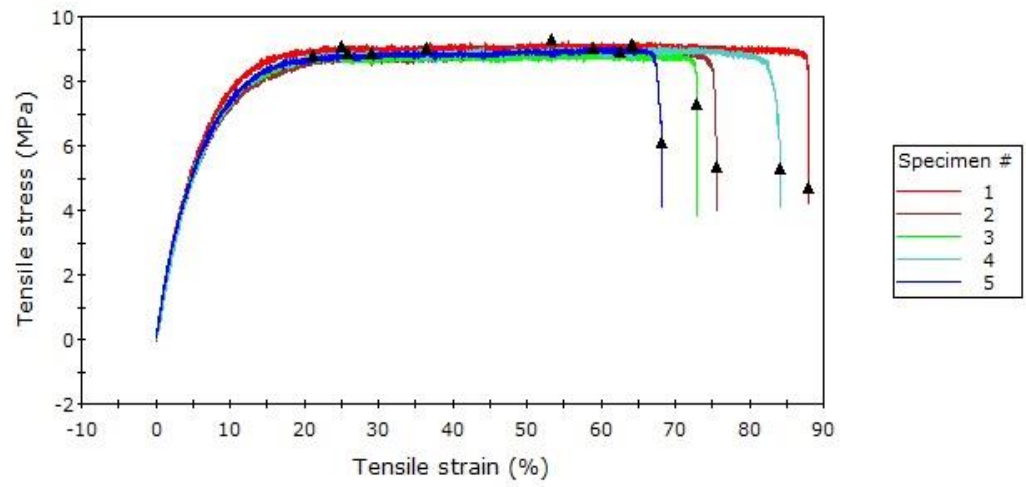

Figure 6. Tensile stress Vs strain for the LLDPE / 5 wt \% EG at 200 P\&F cycles.

The young's modulus for the LLDPE / $10.7 \mathrm{wt} \%$ EG filled nanocomposites showed a good result when comparing with the young's modulus of the pristine LLDPE. But the lower filler concentration (up to 50 cycles) showed approximately equivalent young's modulus to the pristine LLDPE (Fig. 7). It started to gradually increase in the following cycles and reached the maximum of $320 \mathrm{MPa}$ at the 500 cycles. 

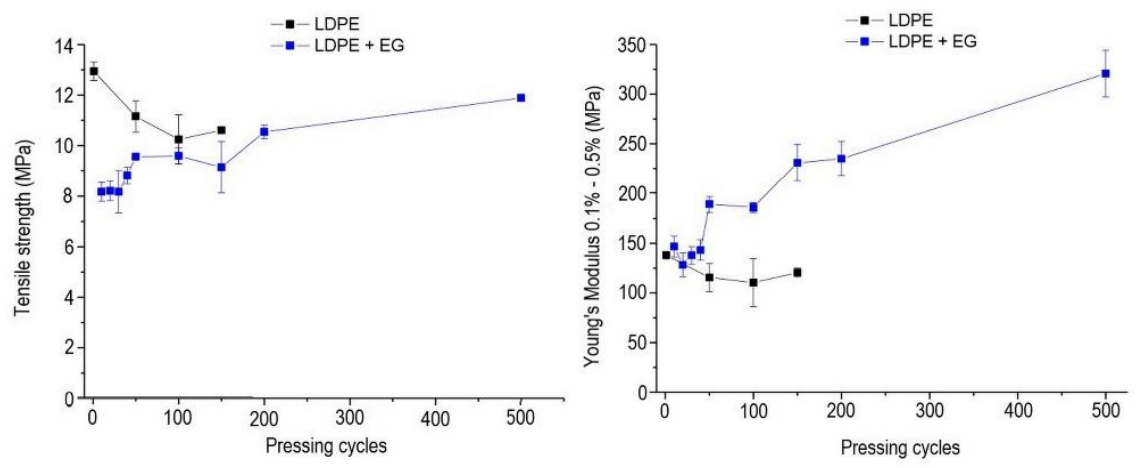

Figure 7. Young's Modulus and tensile strength of the LLDPE / $10.7 \mathrm{wt} \%$ EG as a function of $P \& F$ cycle

Clearly the presence of EG fillers reduces the ductility of the pristine LLDPE, which shows in the necking without break in the tensile test. Tensile failure depends critically on any localized deformation or agglomeration of EG fillers on any particular area of the specimen. The Young's modulus is measured from the initial region of tensile deformation and indicative of the composite value of the constituent stiffness [30]. Comparing the EG filled composites of higher cycles show a higher modulus than that of the unreinforced LLDPE. Fig. 8 shows the variation of reinforcement $(\mathrm{Ec} / \mathrm{Em})$ with $\mathrm{EG}$ content for nanocomposites produced by $\mathrm{P} \& \mathrm{~F}$ technique.

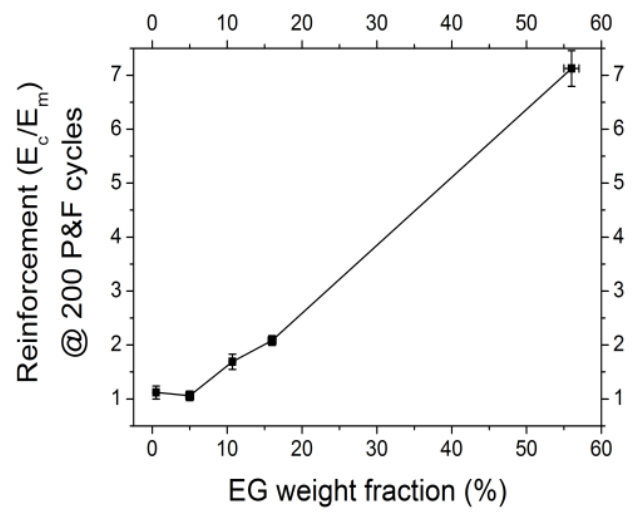

Figure 8. Variation of Reinforcement with EG content 
There is a decrease in the reinforcement (Ec/Em) from $0.5 \mathrm{wt} \%$ to $5 \mathrm{wt} \%$ and that is because of the poor dispersion of the filler content or the presence of defect in tensile tested area. The higher reinforcement is due to the good dispersion of the fillers but it has a more threshold value by adding $56 \mathrm{wt} \%$ of EG. The strong interaction between the EG and LLDPE polymer matrix could result from, the wrinkled surface of extremely thin graphite sheets that is capable of mechanically interlocking with polymer chains.

\subsection{Electrical Characterization}

The conductivity of EG reinforced LLDPE was measured using a two-point resistivity probe system with the minimum limit of $10^{-8} \mathrm{~S} / \mathrm{cm}$. The hot-pressed sample was cut into specimens for testing of $12 \times 8 \times 0.25 \mathrm{~mm}^{3}$. The first two set of samples ( 0.5 and $5 \mathrm{wt} \%$ of EG) does not conduct in all the cycles. The specimen started to conduct when $10.7 \mathrm{wt} \%$ of EG is added to the LLDPE. Therefore, only three sets of samples $(10.7,16,56 \mathrm{wt} \% \mathrm{EG})$ are analysed for the electrical testing.

Conduction in graphene-based composites is usually described in terms of conventional static percolation theory, which is adequate in describing the dynamic process of conductive network formation. When preparing polymer/graphene composites, a high sheer is often applied during mixing in order to disperse EG in polymer matrix, leaving them separated from each other, resulting in low composite conductivity. These well dispersed and separated EGs are however reported to aggregate in the melt given sufficient temperature and time leading to high orders of magnitude improvement in composite conductivity. This means that for the same concentration of EG, a composite can show very different levels of conductivity. For this reason, one should be careful in comparing data reporting conductivity and percolation threshold values. This phenomenon is been termed as Dynamic Percolation. For filler content greater than the percolation threshold, the percolation curve can be fitted by an exponential function (1),

$$
\sigma=\sigma_{o}\left(p-p_{c}\right)^{t},
$$

where $\sigma_{\mathrm{o}}$ is the scaling factor, $\mathrm{p}_{\mathrm{c}}$ is the percolation threshold, $\mathrm{t}$ is the characteristic exponent which depends on the dimensionality of the system ( $\mathrm{t} \sim 2$ for $3 \mathrm{D}$ system; $\mathrm{t} \sim 1.3$ for $2 \mathrm{D}$ system). $\mathrm{p}_{\mathrm{c}}$ is inversely proportional to the particle aspect ratio [31]. This is the reason why conductive nanoparticles like GNPs, CNTs are interesting as fillers.

Fig. 9 shows the variation of the electrical conductivity of LLDPE / 10.7 $\mathrm{wt} \%$ EG nanocomposites as a function of the P\&F cycles. Like most polymers, LLDPE is not electrically conductive and its room temperature electrical resistivity is $3.3 \mathrm{e} 24-3 \mathrm{e} 25 \mu \Omega . \mathrm{cm}$. The addition of EG greatly improved its conductivity with a sharp transition from an electrical insulator to an electrical conductor. The 
percolation threshold value of the conducting composite is about $10.7 \mathrm{wt} \%$ of expanded graphite, which is much higher than the other polymer composites. The percolation threshold for the electrical conductivity depends very much on the geometry of the conductive filler. Filler with a higher aspect ratio such as sheet-like or fibre-like filler, has an advantage in forming a conductive network in polymer matrix than that of filler in either round or ellipse shape [32].

Even though the composite has a high percolation threshold, it was found that the EG particles were increasingly distributed and dispersed in LLDPE with the number of cycles and mainly oriented on the plane of the samples. This find was confirmed by electrical resistivity measurements. A low in -plane resistivity (about $300 \Omega \mathrm{cm}$, corresponding to a conductivity of $0.003 \mathrm{~S} / \mathrm{cm}$, from Fig. 9) was found for nanocomposites prepared between 150 to 500 cycles. For each sample, the resistivity out- of plane was about 10.000 times higher than the in-plane resistivity (Fig. 9).
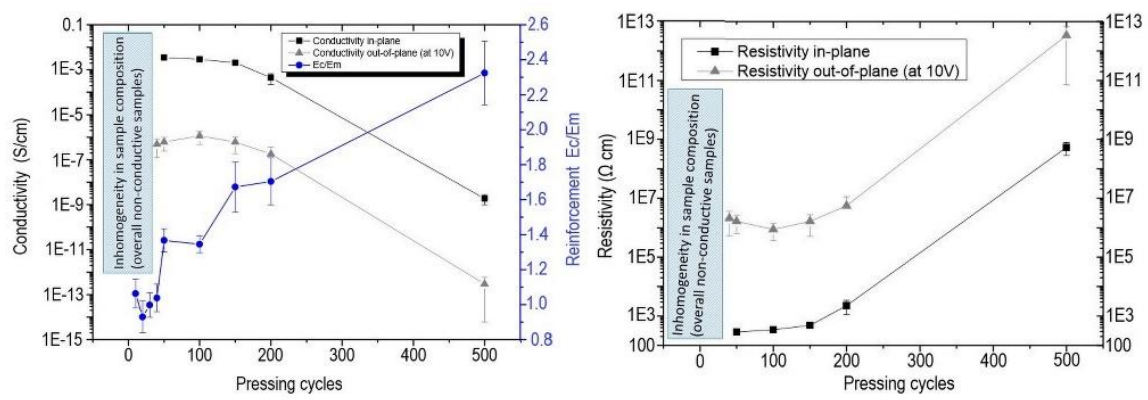

Figure 9. Electrical conductivity and resistivity of LLDPE / 10.7 wt\% EG as a function of $P \& F$ cycles.

The conductivity data collected from the three different wt \% of EG (10.7, 16 and $56 \mathrm{wt} \%$ at 200 cycles) are presented in Fig. 10 and it revealed that at lower filler concentration (below percolation threshold value of $10.7 \%$ ), the small EG particles that tend to aggregate into larger low aspect ratio clusters. It tends to disperse more evenly into the polymer matrix, thereby resulting in fewer particleparticle interactions at a given loading level and consequently leading to lower or no conductivity polymer composite. As the filler concentration is increased beyond the threshold concentration, the conductivity of all LLDPE composites is observed to increase rapidly. 


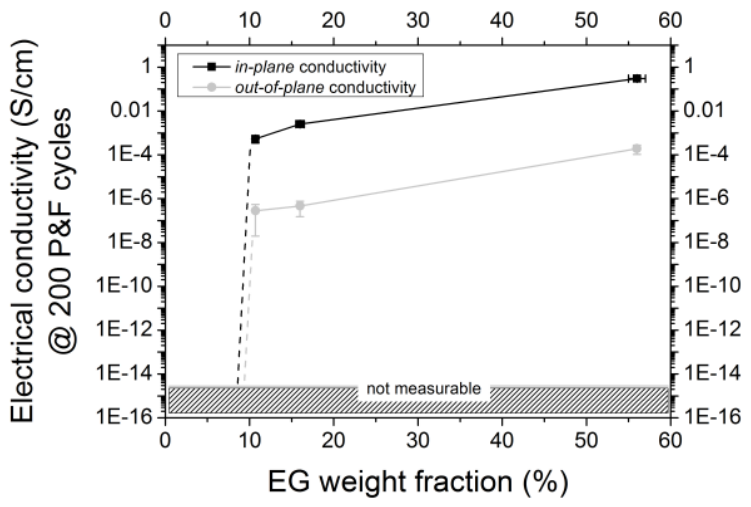

Figure 10. Electrical conductivity of LLDPE / EG composites at 200 cycles for different EG concentration.

Once the electrical percolation has been achieved, the increase in conductivity as a function of filler loading can be also modelled by a simple powerlaw expression (2),

$$
\sigma_{c}=\sigma_{f}\left(\phi-\phi_{c}\right)^{t}
$$

where $\phi$ is the filler volume fraction, $\phi_{\mathrm{c}}$ is the percolation threshold, $\sigma_{\mathrm{f}}$ is the filler conductivity, $\sigma_{\mathrm{c}}$ is the composite conductivity and $t$ is a scaling exponent [33]. The filler need not be in the direct contact for current flow; rather, conduction can take place via tunnelling between thin polymer layers surrounding the filler particles and this tunnelling resistance is the limiting factor in the composite conductivity [33]. Chen et al [34] reported that CNT /epoxy nanocomposites have an electrical percolation threshold as low as approximately $0.0025 \mathrm{wt} \%$, which is by far the lowest, reported for any graphene-based nanocomposites. These exceptional results attribute to the kinetic percolation that most notably arises in composites with a low viscosity during processing. Eventually, it forms a three-dimensional network of filler content that electrically percolates at a much lower loading than possible with well dispersed and randomly oriented fillers. It has been said that a high degree of dispersion may not necessarily yield the lowest electrical percolation [33]. Indeed, the lowest percolation threshold achieved thus far for a graphene-based polymer nanocomposite ( $0.15 \mathrm{wt} \%$ [32]) was observed when the filler was not homogeneously dispersed in the polymer matrix, but rather segregated from the matrix to form a conductive network.

Alignment of the filler also plays a major role in the onset of the electrical percolation. When the EG particles are aligned in the matrix, there are, at least at 
relatively low concentrations ( 0.5 and $5 \mathrm{wt} \%)$, fewer contacts between them and thus the percolation threshold would increase till the composite starts to conduct at higher filler concentrations.

A series of SEM micrographs obtained from the samples of different filler concentration and different cycles are presented in the next section. The SEM images of the LLDPE/EG samples near the threshold concentration (10.7 wt\%) reveal the existence of EG particles exhibiting short and long-range connectivity in the plane and through the thickness of the composite film. The SEM images from the LLDPE/10.7 wt\% EG samples, show that the fibres are primarily, but not exclusively, aligned in the specimen plane throughout the thickness of the sample. Because of this difference in morphological behaviour of the sample, the electrical conductivity also changes its behaviour according to the orientation of the filler content. The in plane and the out of plane current for the LLDPE/10.7 wt\% at different cycles as a function of applied voltage are shown in the Fig. 11.
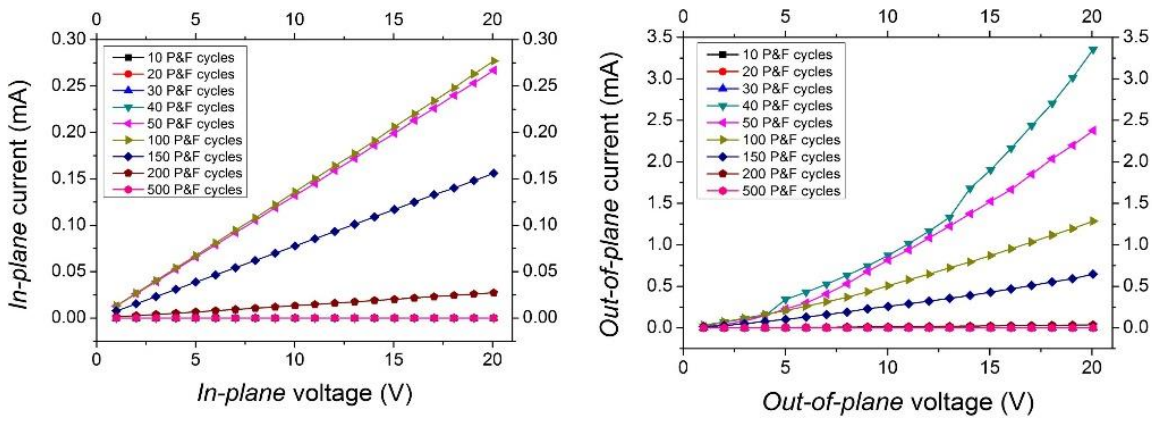

Figure 11. Variation of current vs voltage for in-plane and out-of-plane filler orientation

Dispersion of graphite is also important to the variation of percolation threshold for conductivity transition in the composites. In my experiment, I used long time, intensive pressing and folding (500 cycles) to promote fine dispersion for the composites. The sufficient adsorption of the LLDPE molecular chains onto various pores of the expanded graphite was also the likely factor contributing to good dispersion. If the solution concentration is too high, the higher viscosity may hinder the polymer chains from entering the minor pores of the graphite and thus lead to poor dispersion of graphite flakes in the polymer. Also, the variation of current vs voltage for in -plane and out-of-plane orientation for the filler content above the percolation threshold (i.e., 10.7, 16, 56 wt \% EG) has been shown in the Fig. 12. 

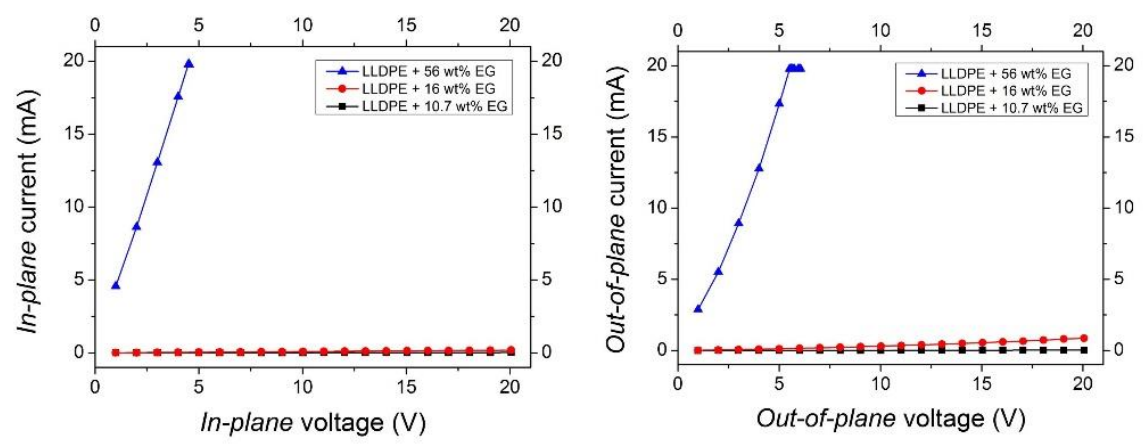

Figure 12. Variation of current vs voltage for in-plane and out-of-plane filler orientation for different filler concentration at 200cycles

\subsection{Morphological Characterization}

As the mechanical and the electrical property enhancements correlate strongly with nanocomposite microstructure, effective characterization of morphology is also important in establishing the structure-property relationships for these composites. SEM studies are perhaps the most common means by which the state of dispersion can be assessed. The expanded graphite used here is a particlebased filler (rather fibre-based filler), as it has the high aspect ratio. The higher aspect ratio platelets are generally found to be beneficial to the mechanical, electrical and thermal properties of the composite material [33]. An exfoliated morphology of EG is thus usually desired as it provides higher aspect ratio platelets relative to stacked or intercalated platelets. The cross-sectional analysis of the composite is also performed using SEM which has been used to evaluate the dispersion of EG as well as to examine the surface for pull -out, possibly giving insight into the strength of interfacial adhesion. Exfoliated graphene-based materials are often compliant and when dispersed in a polymer matrix are typically not observed as a rigid disk, but rather as bent or crumpled platelets as shown in Fig. 13. The cross section of the cryogenically broken sample for different filler content at 200 cycles is shown in the Fig. 14 a, b, c. The images show that the graphene is very well dispersed at the higher concentration and the three-dimensional network of the conductive fillers increase as the filler concentration increase. This leads to the higher percolation content at $10.7 \mathrm{wt} \%$. The analogous images acquired from the LLDPE / EG samples (Fig. 14d) show that the fibres are primarily, but not exclusively, aligned in the specimen plane throughout the thickness of the sample. 
P. K. Rajamani and R. Boros - Acta Technica Jaurinensis, Vol. 11, No. 4, pp. 185-205, 2018
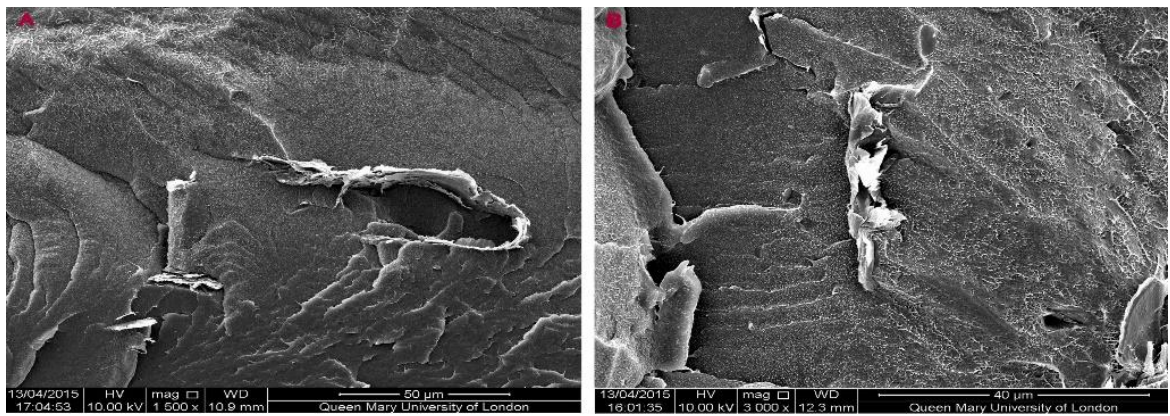

Figure 13. Identified graphite sheets in cryogenically broken sample of LLDPE / 0.5 wt\% EG after A) $30 \mathrm{P \& F}$ cycles B) $200 \mathrm{P} \& \mathrm{~F}$ cycles
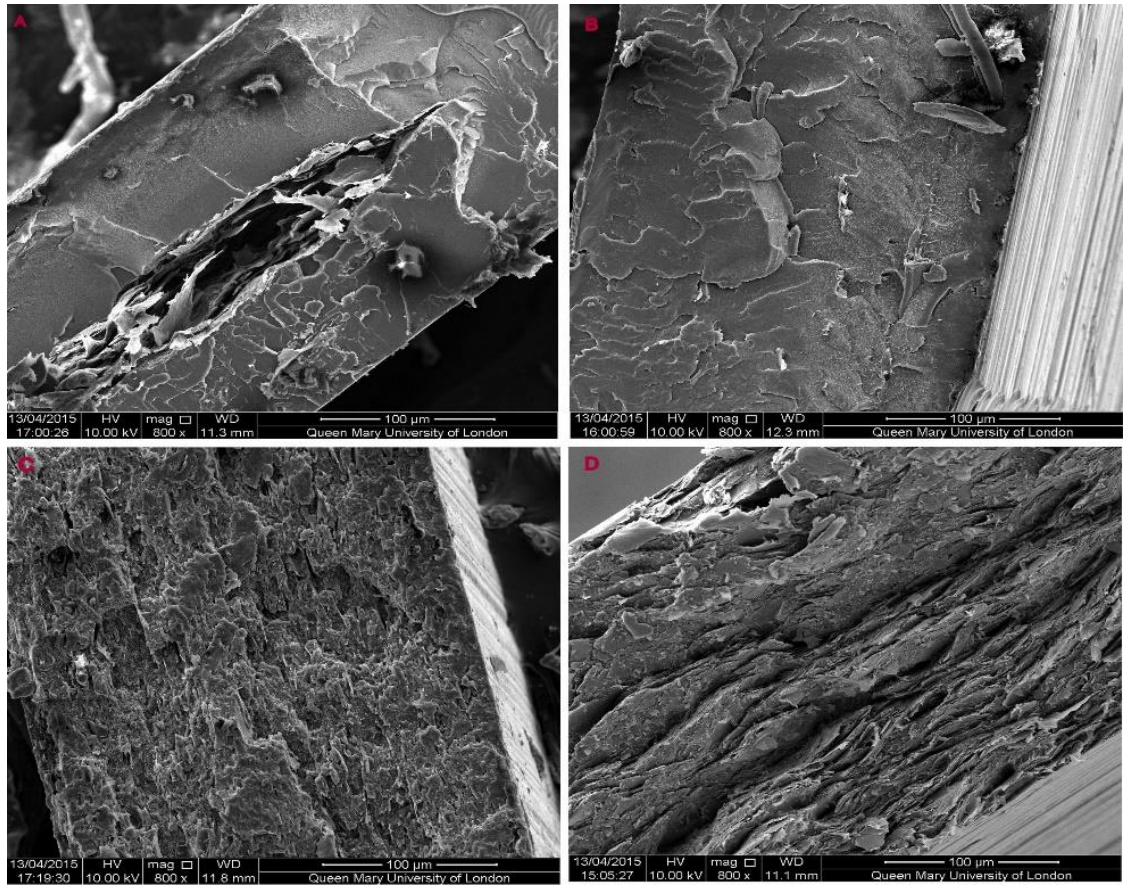

Figure 14. SEM images of cryogenically broken samples of $0.5 \mathrm{wt} \%$ EG content a) 10 cycles b) 200 cycles c) 500 cycles and d) 56 EG wt\% at 200 cycles 


\section{Conclusions}

In this study, we focused on studying the mechanical and the electrical properties of LLDPE/EG nanocomposites with different filler content and different P\&F cycles. Apparently, the EG system demonstrated better properties from a higher aspect ratio, as the filler content increases as well the pressing cycles increases. Some specific conclusions could be drawn as follows,

- The materials studied were fabricated in an alternative top-down production technique called Pressing \& Folding technique and demonstrated the useful mechanical and thermal properties along with the morphological study of different filler content which was processed for different P\&F cycles. Future development of graphite or graphene-based nanocomposites awaits better dispersion of the nano fillers in the polymer matrix and the control of percolation transition in applications.

- Viscosity increased with the addition of graphite fillers. The introduction of EG increased the stiffness of the composites. At $56 \mathrm{wt} \%$, which is considered as the maximum filler loading content in the polymer showed high viscosity than the lower EG content. The difference was attributed to EG's higher area to volume and aspect ratios which promoted a more viscous flow in LLDPE matrix.

- Electrical conductivity for the composites showed a transition from an insulator to a conductor. But it requires a minimum of $10.7 \mathrm{wt} \%$ of EG content to reach the percolation threshold. It was also found that the EG particles were increasingly distributed and dispersed in LLDPE with the number of cycles and mainly oriented on the plane of the samples. Although LLDPE is a good insulator, a low in-plane resistivity was found for nanocomposites prepared with more P \& F cycles (200 cycles). For each sample, the out - of -plane resistivity was about 10.000 times higher than the in-plane resistivity.

- The results from the micro-structures of the prepared samples were studied by analysing the cross sections of the cryogenically broken and mechanically broken (tensile) samples by SEM. It was found that most of the EG particles are mainly oriented on the plane of the samples.

- Both tensile and electrical measurements indicated EG were better filler in the LLDPE. Although overall improvement in mechanical properties was not impressive, but our results clearly confirmed the advantages of using EG by $\mathrm{P} \& \mathrm{~F}$ method to enhance both the electrical conductivity and mechanical strength and stiffness of LLDPE. Therefore, the role of EG as a reinforcement phase was unambiguously established. 


\section{Acknowledgement}

I wish to specially thank the Nanovision and Nanoforce Laboratory at Queen Mary University of London for their technical assistance and valuable support in terms of materials and equipment.

\section{References}

[1] S. Park, R.S. Ruoff, Chemical methods for the production of graphenes, Nature Nanotechnology 4 (4) (2009) pp. 217-224.

DOI: https://doi.org/10.1038/nnano.2009.58

[2] A.K. Geim, K.S. Novoselov, The rise of graphene, Nature Materials 6 (3) (2007) pp. 183-191.

DOI: https://doi.org/10.1038/nmat1849

[3] H. Shioyama, Cleavage of graphite to graphene, Journal of Materials Science Letters 20 (6) (2001) pp. 499-500.

DOI: https://doi.org/10.1023/A:1010907928709

[4] Y. Li, S. Wang, Q. Wang, M. Xing, A comparison study on mechanical properties of polymer composites reinforced by carbon nanotubes and graphene sheet, Composites Part B 133 (2018) pp. 35-41.

DOI: https://doi.org/10.1016/j.compositesb.2017.09.024

[5] V.D. Punetha, S. Rana, H.J. Yoo, A. Chaurasia, J.T. McLeskey Jr, M.S. Ramasamy, N.G. Sahoo, J.W. Cho, Functionalization of carbon nanomaterials for advanced polymer nanocomposites: A comparison study between CNT and graphene, Progress in Polymer Science 67 (2017) pp. 147.

DOI: https://doi.org/10.1016/j.progpolymsci.2016.12.010

[6] H.Yao, H.J. Sue, Preparation of epoxy nanocomposites containing welldispersed graphene nanosheets, Composites Science and Technology 146 (2017) pp. 161-168.

DOI: https://doi.org/10.1016/j.compscitech.2017.04.026 
[7] J.Z. Liang, Q. Du, G.C. Tsui, C.Y. Tang, Tensile properties of graphene nano-platelets reinforced polypropylene composites, Composites Part B 95 (2016) pp. 166-171.

DOI: https://doi.org/10.1016/j.compositesb.2016.04.011

[8] Y. Guo, G. Xu, X. Yang, K. Ruan, T. Ma, Q. Zhang, J. Gu, Y. Wu, H. Liu, Z. Guo, Significantly enhanced and precisely modelled thermal conductivity in polyimide nanocomposites with chemically modified graphene via in situ polymerization and electrospinning-hot press technology, Journal of Materials Chemistry C 6 (12) (2018) pp. 3004-3015. DOI: $\underline{10.1039 / \mathrm{C} 8 \mathrm{TC} 00452 \mathrm{H}}$

[9] Y. Lan, Z. Guo, Electrically conductive thermoplastic polyurethane/ polypropylene nanocomposites with selectively distributed graphene, Polymer 97 (2016) pp. 11-19.

DOI: https://doi.org/10.1016/j.polymer.2016.05.017

[10] Y. Cui, S. Kumar, Gas barrier performance of graphene/polymer nanocomposites, Carbon 98 (2016) pp. 313-333.

DOI: https://doi.org/10.1016/j.carbon.2015.11.018

[11] A.P. Bafana, E.K. Wujcik, Polypropylene nanocomposites reinforced with low weight percent graphene nanoplatelets, Composites Part B: Engineering 109 (2017) pp. 101-107.

DOI: https://doi.org/10.1016/j.compositesb.2016.10.048

[12] J.R. Potts, R.S. Ruoff, Graphene-based polymer nanocomposites. Polymer 52 (1) (2011) pp. 5-25.

DOI: https://doi.org/10.1016/j.polymer.2010.11.042

[13] B.Z. Jang, A. Zhamu, Processing of nanographene platelets (NGPs) and NGP nanocomposites: A review, Journal of Materials Science 43 (15) (2008) pp. 5092-5101.

DOI: https://doi.org/10.1007/s10853-008-2755-2

[14] K. Kalaitzidou, H. Fukushima, L.T. Drzal, A new compounding method for exfoliated graphite-polypropylene nanocomposites with enhanced flexural properties and lower percolation threshold, Composites Science and Technology 67 (2007) pp. 2045-2051.

DOI: https://doi.org/10.1016/j.compscitech.2006.11.014 
[15] W.S. Kang, S.J. Park, Thermal, impact and toughness behaviours of expanded graphite/graphite oxide-filled epoxy composites, Composites Part B: Engineering 94 (2016) pp. 238-244.

DOI: https://doi.org/10.1016/j.compositesb.2016.03.052

[16] G. Wang, Z.Dai, L. Liu, H.Hu, Q. Dai, Z.Zhang, Tuning the interfacial mechanical behaviours of monolayer graphene/PMMA nanocomposites, ACS applied materials \& Interfaces 8 (2016) pp. 22554-22562. DOI: $\underline{10.1021 / \mathrm{acsami} .6 \mathrm{~b} 03069}$

[17] K.Wu, Q.Fu, Largely enhanced thermal and electrical conductivity via constructing double percolated filler network in polypropylene/expanded graphite - Multi-wall carbon nanotubes ternary composites, Composites Science and Technology 130 (2016) pp. 28-35.

DOI: https://doi.org/10.1016/j.compscitech.2016.04.034

[18] K. Gaska, X.Xu, S. Gubanski, R. Kadar, Electrical, Mechanical, and Thermal Properties of LDPE Graphene Nanoplatelets Composites Produced by Means of Melt Extrusion Process. Polymers 9 (1) (2017) pp. 11-23. DOI: https://doi.org/10.3390/polym9010011

[19] A.A. Vasileiou, M. Kontopoulou, A.Docoslis, A noncovalent compatibilization approach to improve the filler dispersion and properties of polyethylene/graphene composites, ACS Applied Material Interfaces 6 (3) (2014) pp. 1916-1925.

DOI: $\underline{10.1021 / \mathrm{am} 404979 \mathrm{~g}}$

[20] H.R. Mamagani, V. Haddadi-Asl, K. Khezri, M.S. Kalajahi, Polystyrenegrafted graphene nanoplatelets with various graft densities by atom transfer radical polymerization from the edge carboxyl groups, RSC Advances 4 (47) (2014) pp. 24439-24452.

DOI: http://dx.doi.org/10.1039/c4ra03451a

[21] S. Roy, X. Tang, T. Das, L. Zhang, Y. Li, S. Ting, X. Hu, C.Y. Yue, Enhanced Molecular Level Dispersion and Interface Bonding at Low Loading of Modified Graphene Oxide To Fabricate Super Nylon 12 Composites, ACS Applied Materials Interfaces 7 (5) (2015) pp. 3142-3151. DOI: $\underline{10.1021 / \mathrm{am} 5074408}$ 
[22] H.Xu, L.X. Gong, X. Wang, L. Zhao, Y.B. Pei, G. Wang, Y.J. Liu, L.B. $\mathrm{Wu}$, J.X. Jiang, L.C. Tang, Influence of processing conditions on dispersion, electrical and mechanical properties of graphene filled silicone rubber composies, Composites Part A: Applied Science and Manufacturing 91 (1) (2016) pp. 53-64.

DOI: https://doi.org/10.1016/j.compositesa.2016.09.011

[23] T. Kuilla, S. Bhadra, D. Yao, N.H. Kim, S. Bose, J.H. Lee, Recent advances in graphene based polymer composites, Progress in Polymer Science 35 (11) (2010) pp. 1350-1375.

DOI: https://doi.org/10.1016/j.progpolymsci.2010.07.005

[24] O.M. Istrate, K.R. Paton, U. Khan, A. O’Neil, A.P. Bell, J.N. Coleman, Reinforcement in melt-processed polymer-graphene composites at extremely low graphene loading level, Carbon 78 (2014) pp. 243-249. DOI: https://doi.org/10.1016/j.carbon.2014.06.077

[25] L.C. Tang, Y.J. Wan, D. Yan, Y.B. Pei, L. Zhao, Y.B. Li, L.B. Wu, J.X. jiang, G.Q. Lai, The effect of graphene dispersion on the mechanical properties of graphene/epoxy composites, Carbon 60 (2013) pp. 16-27. DOI: https://doi.org/10.1016/j.carbon.2013.03.050

[26] X. Zhang, X.Fan, H. Li, C. Yan, Facile preparation route for graphene oxide reinforced polyamide 6 composites via in situ anionic ring-opening polymerization, Journal of Materials Chemistry 22 (45) (2012) pp. 2408124091.

DOI: $\underline{10.1039 / C 2 J M 34243 J}$

[27] H. Wang, Q. Hao, X. Yang, L. Lu, X. Wang, Effect of Graphene Oxide on the Properties of Its Composite with Polyaniline, ACS Applied Materials Interfaces 2 (3) (2010) pp. 821-828.

DOI: http://dx.doi.org/10.1021/am900815k

[28] H. Hu, G. Zhang, L. Xiao, H. Wang, Q. Zhang, Z. Zhao, Preparation and electrical conductivity of graphene/ultrahigh molecular weight polyethylene composites with a segregated structure, Carbon 50 (12) (2012) pp. 45964599.

DOI: https://doi.org/10.1016/j.carbon.2012.05.045 
[29] L. Hu, J. Tu, S. Jiao, J. Hou, H. Zhu and D. J. Fray, In situ electrochemical polymerization of a nanorod-PANI-Graphene composite in a reverse micelle electrolyte and its application in a supercapacitor, Physical Chemistry Chemical Physics 14 (45) (2012) pp. 15652-15656. DOI: http://dx.doi.org/10.1039/c2cp42192e

[30] W. Zheng, X. Lu, S.C. Wong, Electrical and mechanical properties of expanded graphite-reinforced high-density polyethylene, Journal of Applied Polymer Science 91 (5) (2004) pp. 2781-2788.

DOI: https://doi.org/10.1002/app.13460

[31] D. Stauffer, A. Aharony, Introduction of Percolation Theory, $2^{\text {nd }}$ Edition, Taylor and Francis, London, 1985.

[32] Y.S. Wang, M.A. O'Gurkis, J.T. Lindt, Electrical properties of exfoliatedgraphite filled polyethylene composites, Polymer Composites 7 (5) (1986) pp. 349-354.

DOI: https://doi.org/10.1002/pc.750070512

[33] M. Zhang, Y. Li, Z. Su, G. Wei, Recent advances in the synthesis and applications of graphene-polymer nanocomposites, Polymer Chemistry 6 (34) (2015) pp. 6107-6124.

DOI: https://doi.org/10.1039/C5PY00777A

[34] X.M. Chen, J.W. Shen, W.Y. Huang, Novel electrically conductive polypropylene/graphite nanocomposites, Journal of Materials Science Letters 21 (3) (2002) pp. 213-214.

DOI: http://dx.doi.org/10.1023/A:1014708808230 